\title{
Growth of GaN@InGaN Core-Shell and Au-GaN Hybrid Nanostructures for Energy Applications
}

\author{
Tevye Kuykendall, ${ }^{1}$ Shaul Aloni, ${ }^{1}$ Ilan Jen-La Plante, ${ }^{2}$ and Taleb Mokari ${ }^{1}$ \\ ${ }^{1}$ The Molecular Foundry, Materials Science Division, Lawrence Berkeley National Laboratory, 1-Cyclotron Road, \\ Berkeley, CA 94720, USA \\ ${ }^{2}$ Department of Chemistry, University of California at Berkeley, 1-Cyclotron Road, Berkeley, CA 94720, USA
}

Correspondence should be addressed to Taleb Mokari, tmokari@lbl.gov

Received 2 April 2009; Accepted 25 May 2009

Recommended by Mohamed Sabry Abdel-Mottaleb

We demonstrated a method to control the bandgap energy of GaN nanowires by forming GaN@InGaN core-shell hybrid structures using metal organic chemical vapor deposition (MOCVD). Furthermore, we show the growth of Au nanoparticles on the surface of $\mathrm{GaN}$ nanowires in solution at room temperature. The work shown here is a first step toward engineering properties that are crucial for the rational design and synthesis of a new class of photocatalytic materials. The hybrid structures were characterized by various techniques, including photoluminescence (PL), energy dispersive $\mathrm{x}$-ray spectroscopy (EDS), transmission and scanning electron microscopy (TEM and SEM), and x-ray diffraction (XRD).

Copyright ( $) 2009$ Tevye Kuykendall et al. This is an open access article distributed under the Creative Commons Attribution License, which permits unrestricted use, distribution, and reproduction in any medium, provided the original work is properly cited.

\section{Introduction}

The optical, electrical, and chemical properties of semiconductor nanostructures have been studied intensively in the last two decades. Semiconductor nanostructures have been shown to have unique properties compared to the bulk. Due to their large surface to volume ratio, effects at the surfaces, and/or interfaces in multicomponent nanomaterials, have been shown to strongly influence the properties of the materials. By controlling the size, shape, and composition of these materials, the energy levels within the materials can be altered in a way that may offer better control over exciton creation, charge separation, recombination, and transport. With the increasing push to find clean energy alternatives to fossil fuels, many recent studies have been exploring the potential use of nanomaterials for energy conversion. Solar energy is possibly one of the cleanest and most abundant sources of renewable energy. Photocatalysis has been shown to be one of the most promising areas of solar energy conversion and is the focus of this research.

Many semiconductors that fulfill the requirements of being a good substrate for photocatalytic reactions possess bandgaps that are too large for solar applications. For example: $\mathrm{GaN}$ has been intensively used for water splitting and other photocatalysis reactions but possesses a wide bandgap $(3.4 \mathrm{eV})$, which is not capable of absorbing light over the majority of the solar spectrum. The need to increase the absorption efficiency of photocatalytic materials in the visible portion of the solar spectrum is one of the major challenges to be addressed.

New "engineered" nanomaterials may offer unique solutions critical for the harvesting of solar radiation and conversion to electrical and chemical energy. The assembly of two or more nanostructured components with precise control over the size, shape, composition, and spatial orientation is desirable not only for the combination of different functionalities, but also for advanced properties that can arise independent of the single-component materials. Engineering hybrid multi component nanostructures draws on the vast array of synthetic techniques now at our disposal to assemble nanocrystals with very different properties; a semiconductor nanocrystal can be combined with a metal in such a way that the hybrid structure can be tailored to a specific application.

$\mathrm{In}_{\mathrm{x}} \mathrm{Ga}_{1-x} \mathrm{~N}$ has been shown to be a good candidate for solar energy conversion since the material forms a solid solution with a bandgap that can be tuned from $3.4 \mathrm{eV}$ to 


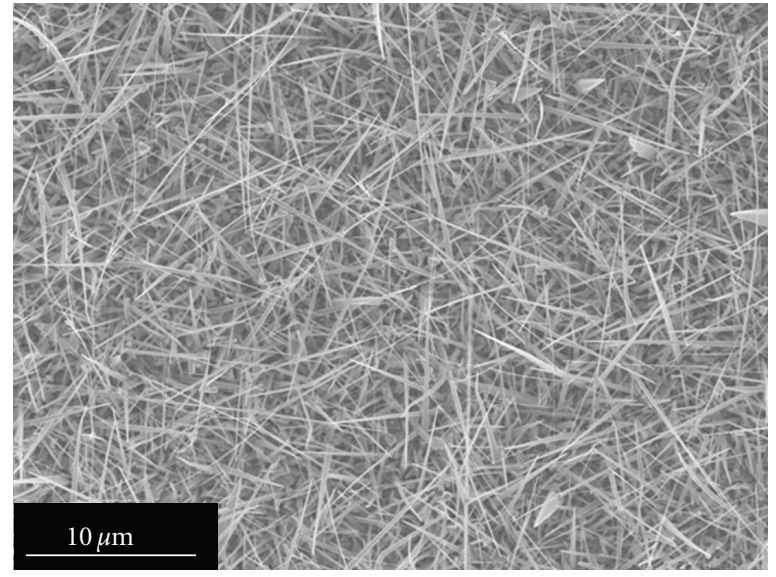

(a)

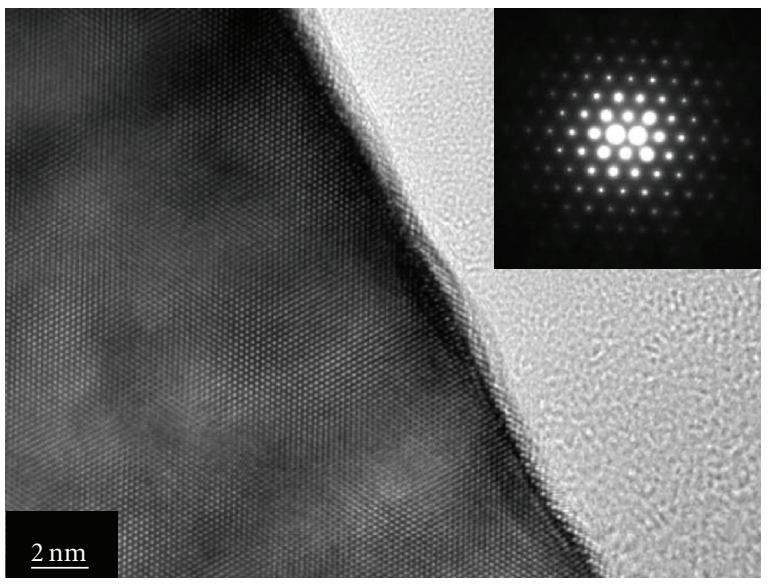

(c)

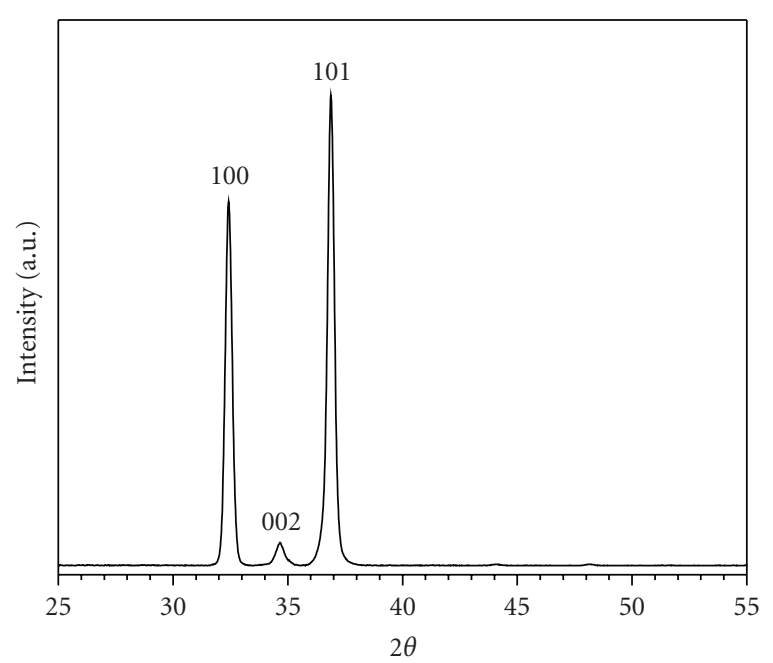

(b)

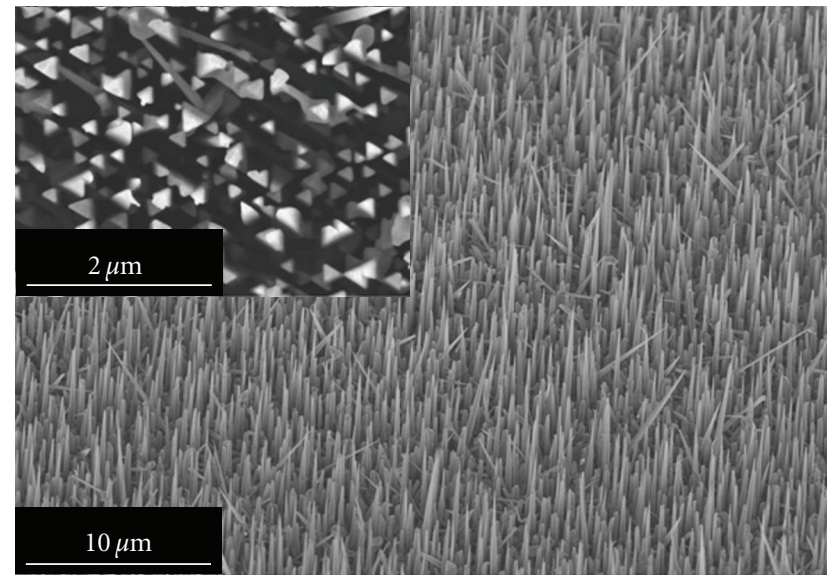

(d)

FIgURE 1: (a) SEM image of GaN nanowires grown with $\mathrm{NiNO}_{3}$ catalyst on c- $\mathrm{Al}_{2} \mathrm{O}_{3}$ substrate. The wires have random orientation with diameters from 50 to $500 \mathrm{~nm}$, and lengths on the order of 10 to $20 \mu \mathrm{m}$. (b) XRD of the GaN nanowires indicates that it has the wurtzite crystal structure. The peaks shown correspond to the (100), (002), and (101) planes from left to right. (c) TEM phase contrast image of a single GaN nanowire oriented along the [001] zone axis. The wires length extends along the [210] direction. The inset shows the electron diffraction pattern, indicating that the wires are single crystalline. (d) SEM showing vertically aligned GaN nanowires on a-LiAlO $\mathrm{G}_{2}$ tilted $30^{\circ}$. The inset shows the triangular cross-section of the nanowires from the top-down view.

$0.9 \mathrm{eV}$ by altering the alloy composition from $x=0$ to $1[1]$. There has been considerable difficulty, however, in producing high-quality InGaN materials with indium concentrations greater than $x \approx 0.2$ due to a large miscibility gap [2]. At high indium concentrations, alloy decomposition, indium segregation, and strain related cracking are often observed. Metalorganic chemical vapor deposition (MOCVD) is the most widely commercially used technique for growing $\mathrm{GaN}$ and InGaN films. While full composition tunability of $\mathrm{In}_{\mathrm{x}} \mathrm{Ga}_{1-x} \mathrm{~N}$ nanowires (with $x=0$ to 1 ) has recently been demonstrated using chloride precursors [3], the direct growth of InGaN nanowires using MOCVD remains elusive. In the chloride approach, relaxation of strain due to the small dimensions of nanowires and low growth temperatures was suggested to be critical in overcoming the miscibility gap. This idea was strengthened by theoretical calculations that modeled indium incorporation as a function of strain and wire diameter [4]. A similar relaxation of strain may be possible in core-shell geometry by reducing the dimensions of the epitaxial interface and may lead to higher indium content alloys.

Here we show that a GaN@InGaN core-shell morphology can be accomplished using MOCVD and will allow us to tune the bandgap of the nanowires to absorb solar radiation in the visible. To achieve this, single-crystalline gallium nitride $\mathrm{GaN}$ nanowire cores were grown $[5,6]$ and subsequently coated with InGaN shells [7]. Trimethylgallium (TMG), trimethylindium, and ammonia were used as the Ga, In, and $\mathrm{N}$ precursors, respectively. The $1 \mathrm{D}$ nanowire growth was achieved using the vapor-liquid-solid (VLS) mechanism [8], with gold or nickel as the catalyst. Using $\mathrm{Ni}\left(\mathrm{NO}_{3}\right)$ as the catalyst's precursor on a c-plane sapphire substrate yielded 


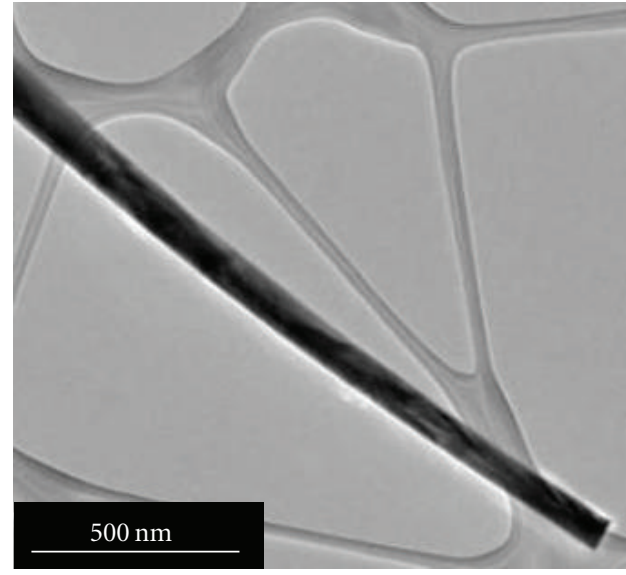

(a)

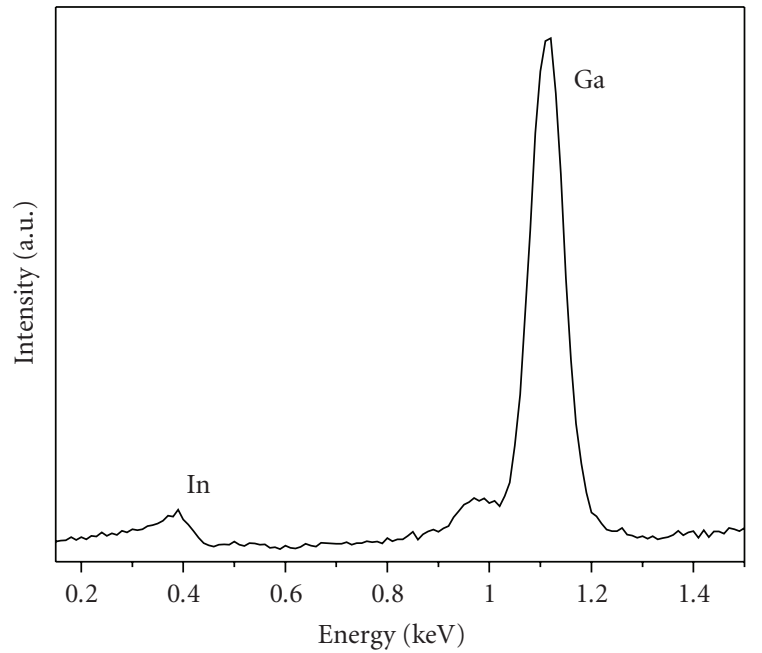

(b)

Figure 2: (a) TEM image showing the morphology of the GaN@InGaN core-shell nanowires. (b) Energy dispersive X-ray spectroscopy shows peaks from the In shell and the Ga shell indicating the presence of Indium in the shell of the GaN@InGaN core-shell heterostructure.

GaN nanowire arrays with no apparent preferred orientation with respect to the substrate. Scanning electron microscopy (SEM) images of the wires are shown in Figure 1(a). The wires generally have triangular cross-sections with widths from 50 to $500 \mathrm{~nm}$ and lengths on the order of 10 to $30 \mu \mathrm{m}$. The X-ray diffraction pattern shown in Figure 1(b) confirms that the wires are $\mathrm{GaN}$ and have the wurtzite crystal structure with lattice constants $a=0.319 \mathrm{~nm}$ and $c=0.514 \mathrm{~nm}$. The transmission electron microscopy (TEM) phase contrast image shown in Figure 1(c), and electron diffraction pattern (inset) confirms that the wires are single crystalline. The images, taken down the [001] zone axis, show that the wire's length extends in the [210] direction. TEM taken for many wires indicates that this is the primary growth direction. The optical properties of the wires will be discussed below.

To improve the orientation and uniformity of the wires we have chosen a substrate that closely matches the preferred wire growth direction [6]. On this substrate the use of gold as the catalyst further improves the wire's uniformity and alignment. The tertiary structure offered by controlling the growth direction and uniformity will provide a better template for later film overgrowth, and particle decoration. An SEM image of GaN wires grown on a- $\mathrm{LiAlO}_{2}$ is shown in Figure 1(d) with the substrate tilted $30^{\circ}$ from normal. The crystallographic orientation along the wires length remained in the [210] direction. However, when $\mathrm{LiAlO}_{2}$ was used, the wires grew vertically and perpendicular to the substrate. Figure 1(d) (inset) is taken from the top-down view, and it clearly shows the triangular cross-sections of the nanowires.

The core-shell wires were synthesized by first growing GaN nanowires (as discussed previously), followed by thinfilm deposition of InGaN at reduced temperatures. TEM images of the nanowires (Figure 2(a)) show that the InGaN shell is a smooth and continuous coating. Energy dispersive X-ray spectroscopy (EDS), shown in Figure 2(b), shows the presence of indium and gallium.
Photoluminescence (PL) spectra were taken for both as-made GaN and core-shell GaN@InGaN nanowires. PL was carried out on individual wires to clearly demonstrate that the emission is from the nanowires and not from a film on the underlying substrate. The PL spectra in Figure 3(a) are of single GaN and GaN@InGaN core-shell nanowires dispersed on glass cover slips. The wires were excited with a $349 \mathrm{~nm}$ Q-switched diode laser operating at $6 \mathrm{kHz}$, focused down to $\sim 5 \mu \mathrm{m}$ spot. The $\mathrm{GaN}$ nanowire showed a peak emission at $384 \mathrm{~nm}(3.2 \mathrm{eV})$. Importantly, the well-known defect induced yellow emission band is not observed, indicating that the high optical quality of these nanowires. The narrow peak observed at $349 \mathrm{~nm}$ is due to the excitation laser. PL spectra of several GaN@InGaN core-shell wires, taken from samples grown at different temperatures, show that the wavelength can be tuned from $380 \mathrm{~nm}$ to $550 \mathrm{~nm}$. CCD images collected from the nanowires shown in Figure 3(b) indicate the color and approximate size of the wires. A plot of emission energy versus concentration is shown in Figure 3(c). The wavelengths observed here correspond to approximate indium concentrations of 0,18 , and 25 percent indium. The presence of two peaks on the right-most spectrum (Figure 3(a)) indicates that there may be some spinodal decomposition or phase segregation of the InGaN shell at higher indium concentrations. Further work will hopefully yield even higher indium concentrations.

There has been significant interest in growth of metals on the surface of nanorods and wires to facilitate charge transfer. A number of approaches have been used to grow metals on semiconductors through reduction, physical deposition, or photochemistry to give $\mathrm{Au}$ and $\mathrm{Ag}$ on $\mathrm{ZnO}[9,10]$, Co and $\mathrm{Au}$ on $\mathrm{TiO}[11,12]$, and others $[13,14]$. Here, we describe our progress on the development of metal-semiconductor heterojunctions focused on GaN nanowires interfaced with Au nanoparticles as shown in Figure 4. Figure 4(a) presents the GaN nanowires before they were introduced to the gold 


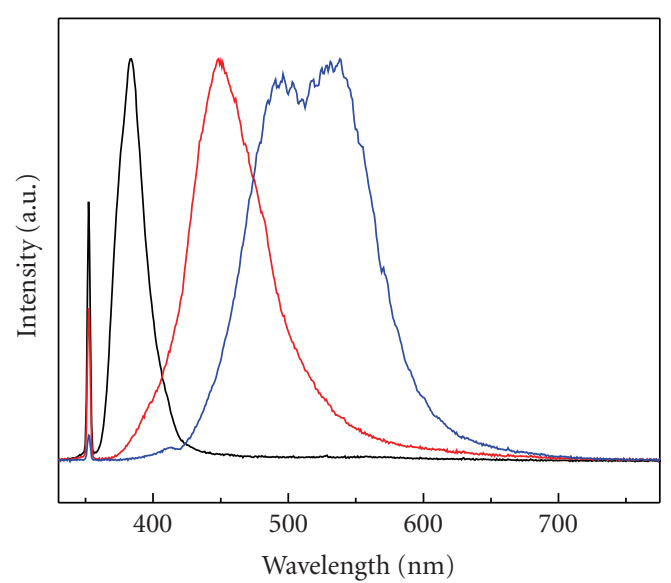

(a)

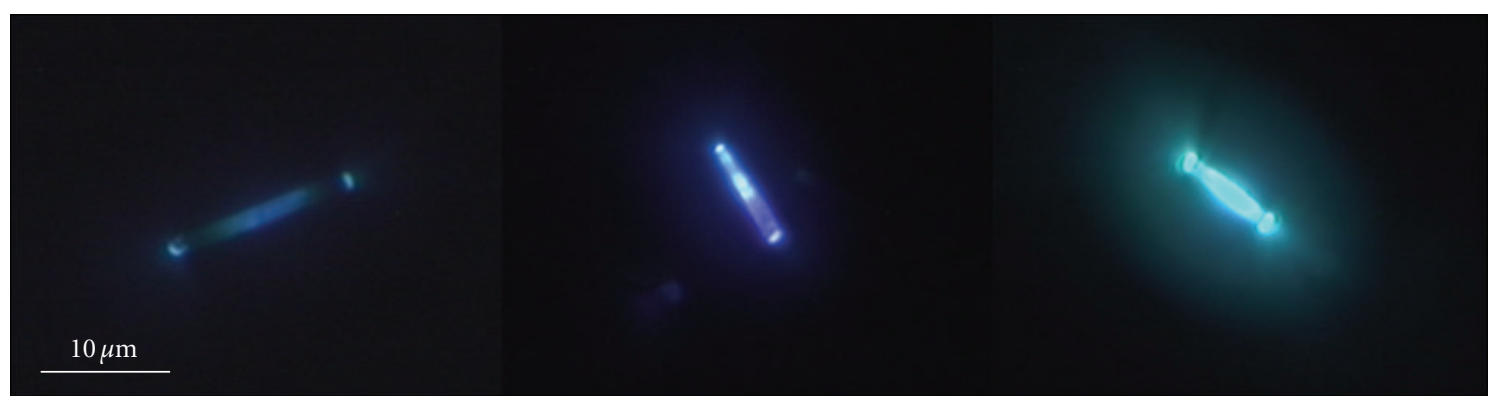

(b)

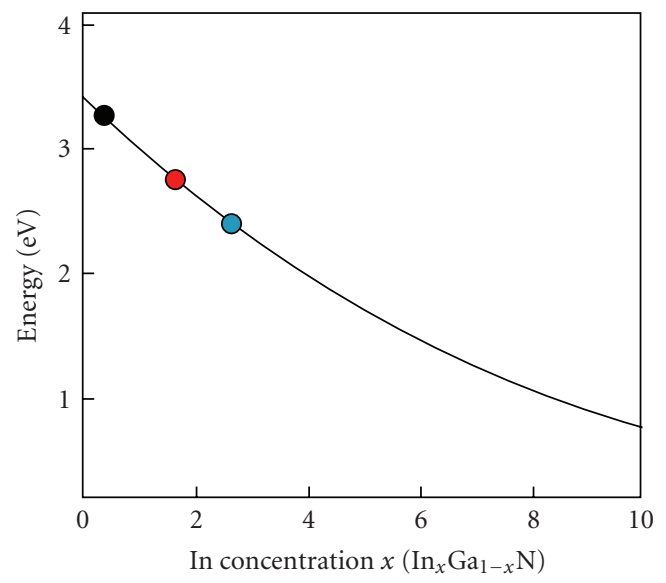

(c)

Figure 3: Photoluminescence of individual wires are shown. (a) PL spectra from left to right with peaks around 380, 450, and $525 \mathrm{~nm}$. The images in (b) are the corresponding wires from the PL spectra in (a) arranged from left to right. (c) A plot of concentration versus bandgap energy shows the approximate concentration of the wire shells.

solution. A facile method for the growth of Au on the tips of CdSe nanorods was developed by one of the co-authors of this paper based on the reduction of Au by dodecylamine in the presence of didodecyldimethylammonium bromide (DDAB) at room temperature. The method was used here to grow gold on the GaN nanowires and is described in the experimental section [15]. The results of the growth are shown in Figure 4(b). The gold particles have an average diameter of $\sim 30 \mathrm{~nm}$. The composition of the hybrid wires (shown in Figure 4(b)) was characterized by EDS and clearly shows a peak that confirms the Au growth.

Conclusion. We demonstrated a method to engineer bandgap by growing $\mathrm{GaN} / \mathrm{InGaN}$ core-shell structures. The bandgap energy was tuned from $380 \mathrm{~nm}$ to $550 \mathrm{~nm}$, which could improve the absorption efficiency. Then, we succeeded to grow catalytic metal $(\mathrm{Au})$ on the surface of the $\mathrm{GaN}$ nanowires, an important hybrid structure for photocatalysis. 


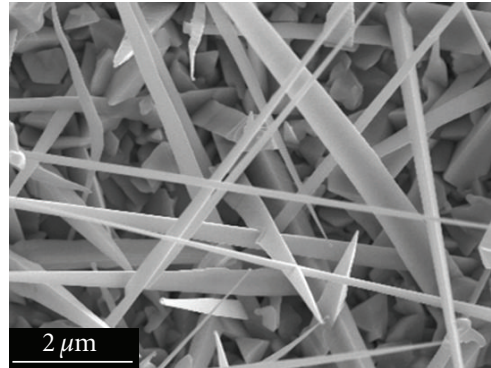

(a)

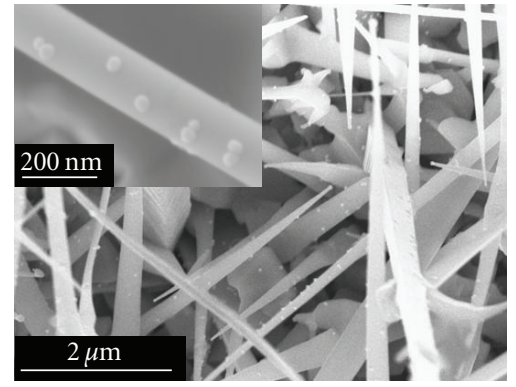

(b)

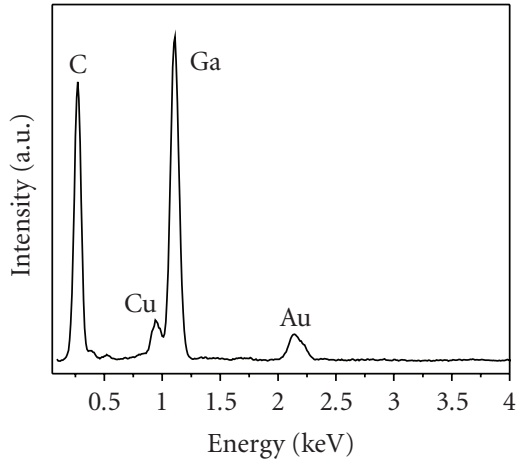

(c)

FIGURE 4: SEM images taken (a) before and (b) after growth of gold nanoparticles on GaN nanowires. (b) Gold nanoparticles can clearly be seen to decorate the surfaces of the GaN nanowires, the inset shows a close up of a single wire with multiple particles attached. (c) EDS of the particles taken by TEM confirms that they are gold.

\section{Experimental}

GaN and GaN@InGaN core-shell nanowire growth was carried out in a Thomas Swan, Showerhead $3 \times 2$ MOCVD cold-wall reactor. The growth substrates were prepared by drop-casting $40 \mu \mathrm{L}$ of $0.2 \mathrm{M}$ solution of $\mathrm{NiNO}_{3}$ in ethanol onto $1 \mathrm{~cm}^{2}$ c-plane sapphire wafer. For $\mathrm{LiAlO}_{2}$ substrates, $3 \mu \mathrm{L}$ of $5: 1$ ratio $\mathrm{B}$. B. International $10 \mathrm{~nm}$ gold colloid to ethanol was drop-cast onto $1 \mathrm{~cm}^{2}$ wafers. Pure GaN nanowires were grown for 10 minutes at $\sim 825^{\circ} \mathrm{C}$. The reactive and carrier gas flows were $600,41,200$, and $400 \mathrm{sccm}$ for ammonia, TMG, hydrogen, and nitrogen. The TMG bubbler was kept at $5^{\circ} \mathrm{C}$, and the reactor pressure was 140 Torr. For core-shell wires, the same conditions were used for GaN nanowires with a subsequent step for InGaN deposition. The InGaN shells were grown for $\sim 15$ minutes with temperatures from $700-750^{\circ} \mathrm{C}$. The reactive and carrier gas flows were 5000, 1, 190, and $1000 \mathrm{sccm}$ for ammonia, TMG, TMI, and nitrogen. The TMI bubbler was kept at $18^{\circ} \mathrm{C}$, and the reactor pressure was 400 Torr.

PL was acquired using a WITec Alpha $300 \mathrm{~S}$ system equipped with a Crystalaser, $349 \mathrm{~nm}$ Q-switched diode laser operating at $6 \mathrm{kHz}$. The laser spot was focused on the nanowire with a spot-size of $\sim 5 \mu \mathrm{m}$. The PL emission was collected through a second objective from an area of $<300 \mathrm{~nm}$ and passed through a fiber to an Ocean Optics QE65000 spectrometer.

SEM and EDS were acquired using a Zeiss Gemini Ultra-55 Analytical Scanning Electron Microscope with an acceleration voltage of $5 \mathrm{kV}$, equipped with an EDAX EDS detector. TEM and EDS were acquired using a JEOL 2100F $200 \mathrm{kV}$ Field-Emission Analytical Transmission Electron Microscope. XRD was acquired using a Bruker AXS D8 Discover GADDS Diffractometer, with a $\mathrm{Cu}-\mathrm{K} \alpha$ source.

\section{Growth of Gold Nanoparticles on the GaN Nanowires}

A stock solution was prepared with $3 \mathrm{~mL}$ toluene, $12 \mathrm{mg}$ gold trichloride, $40 \mathrm{mg}$ didodecyldimethylammonium bromide,
$75 \mathrm{mg}$ dodecylamine and was sonicated for about 20 minutes until the solution changed color to yellow. $1 \mathrm{~mL}$ of this stock solution was added dropwise to the substrate $(4 \mathrm{~mm} \times$ $4 \mathrm{~mm}, \mathrm{Al}_{2} \mathrm{O}_{3}$ ) with $\mathrm{GaN}$ in a vial with $1 \mathrm{~mL}$ of toluene. The final solution was stirred in the vial with the substrate bound GaN and allowed to reduce for 72 hours.

\section{Acknowledgments}

Thanks to Adam Schwartzberg for PL spectroscopy. The work at the Molecular Foundry was supported by the Office of Science, Office of Basic Energy Sciences, of the U.S. Department of Energy under Contract no. DE-AC02$05 \mathrm{CH} 11231$.

\section{References}

[1] J. Wu, W. Walukiewicz, K. M. Yu, et al., "Superior radiation resistance of $\operatorname{In}_{1-x} \mathrm{Ga}_{x} \mathrm{~N}$ alloys: full-solar-spectrum photovoltaic material system," Journal of Applied Physics, vol. 94, no. 10, pp. 6477-6482, 2003.

[2] F. A. Ponce, S. Srinivasan, A. Bell, et al., "Microstructure and electronic properties of InGaN alloys," Physica Status Solidi B, vol. 240, no. 2, pp. 273-284, 2003.

[3] T. Kuykendall, P. Ulrich, S. Aloni, and P. Yang, "Complete composition tunability of InGaN nanowires using a combinatorial approach," Nature Materials, vol. 6, no. 12, pp. 951-956, 2007.

[4] H. J. Xiang, S.-H. Wei, J. L. F. Da Silva, and J. Li, "Strain relaxation and band-gap tunability in ternary $\operatorname{In}_{x} \mathrm{Ga}_{1-x} \mathrm{~N}$ nanowires," Physical Review B, vol. 78, no. 19, Article ID 193301, 4 pages, 2008.

[5] E. A. Stach, P. J. Pauzauskie, T. Kuykendall, J. Goldberger, R. He, and P. Yang, "Watching GaN nanowires grow," Nano Letters, vol. 3, no. 6, pp. 867-869, 2003.

[6] T. Kuykendall, P. J. Pauzauskie, Y. Zhang, et al., "Crystallographic alignment of high-density gallium nitride nanowire arrays," Nature Materials, vol. 3, no. 8, pp. 524-528, 2004.

[7] F. Qian, S. Gradečak, Y. Li, C.-Y. Wen, and C. M. Lieber, "Core/multishell nanowire heterostructures as multicolor, 
high-efficiency light-emitting diodes," Nano Letters, vol. 5, no. 11, pp. 2287-2291, 2005.

[8] Y. Wu and P. Yang, "Direct observation of vapor-liquid-solid nanowire growth," Journal of the American Chemical Society, vol. 123, no. 13, pp. 3165-3166, 2001.

[9] V. Subramanian, E. E. Wolf, and P. V. Kamat, "Green emission to probe photoinduced charging events in $\mathrm{ZnO}-\mathrm{Au}$ nanoparticles. Charge distribution and Fermi-level equilibration," Journal of Physical Chemistry B, vol. 107, no. 30, pp. 74797485, 2003.

[10] C. Pacholski, A. Kornowski, and H. Weller, "Site-specific photodeposition of silver on $\mathrm{ZnO}$ nanorods," Angewandte Chemie International Edition, vol. 43, no. 36, pp. 4774-4777, 2004.

[11] P. V. Kamat, M. Flumiani, and A. Dawson, "Metal-metal and metal-semiconductor composite nanoclusters," Colloids and Surfaces A, vol. 202, no. 2-3, pp. 269-279, 2002.

[12] M. Casavola, V. Grillo, E. Carlino, et al., "Topologically controlled growth of magnetic-metal-functionalized semiconductor oxide nanorods," Nano Letters, vol. 7, no. 5, pp. 13861395, 2007.

[13] D. V. Talapin, H. Yu, E. V. Shevchenko, A. Lobo, and C. B. Murray, "Synthesis of colloidal PbSe/PbS core-shell nanowires and $\mathrm{PbS} / \mathrm{Au}$ nanowire-nanocrystal heterostructures," Journal of Physical Chemistry C, vol. 111, no. 38, pp. 14049-14054, 2007.

[14] J. Yang, H. I. Elim, Q. Zhang, J. Y. Lee, and W. Ji, "Rational synthesis, self-assembly, and optical properties of $\mathrm{PbS}-\mathrm{Au}$ heterogeneous nanostructures via preferential deposition," Journal of the American Chemical Society, vol. 128, no. 36, pp. 11921-11926, 2006.

[15] T. Mokari, E. Rothenberg, I. Popov, R. Costi, and U. Banin, "Selective growth of metal tips onto semiconductor quantum rods and tetrapods," Science, vol. 304, no. 5678, pp. 1787-1790, 2004. 


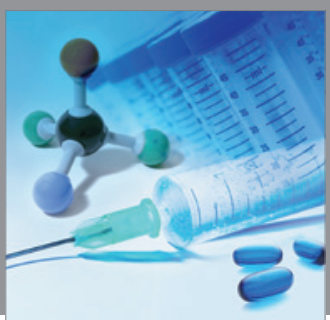

International Journal of

Medicinal Chemistry

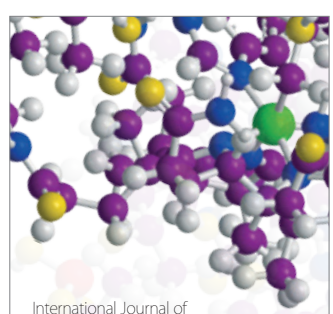

Carbohydrate Chemistry

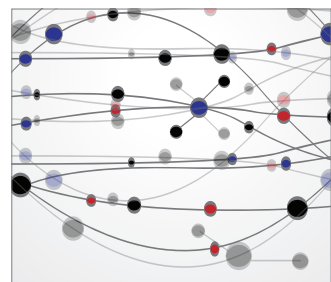

The Scientific World Journal
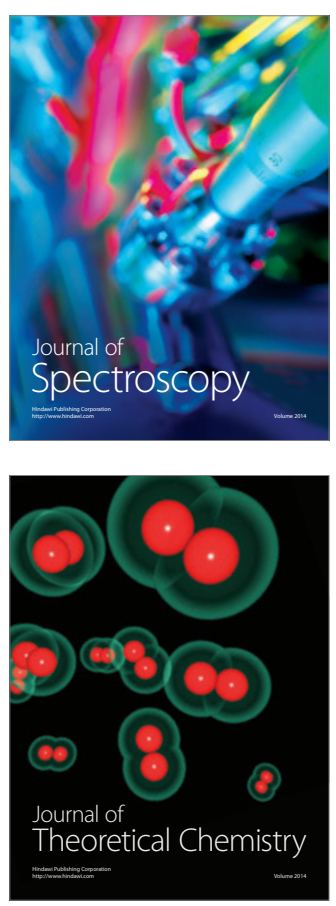
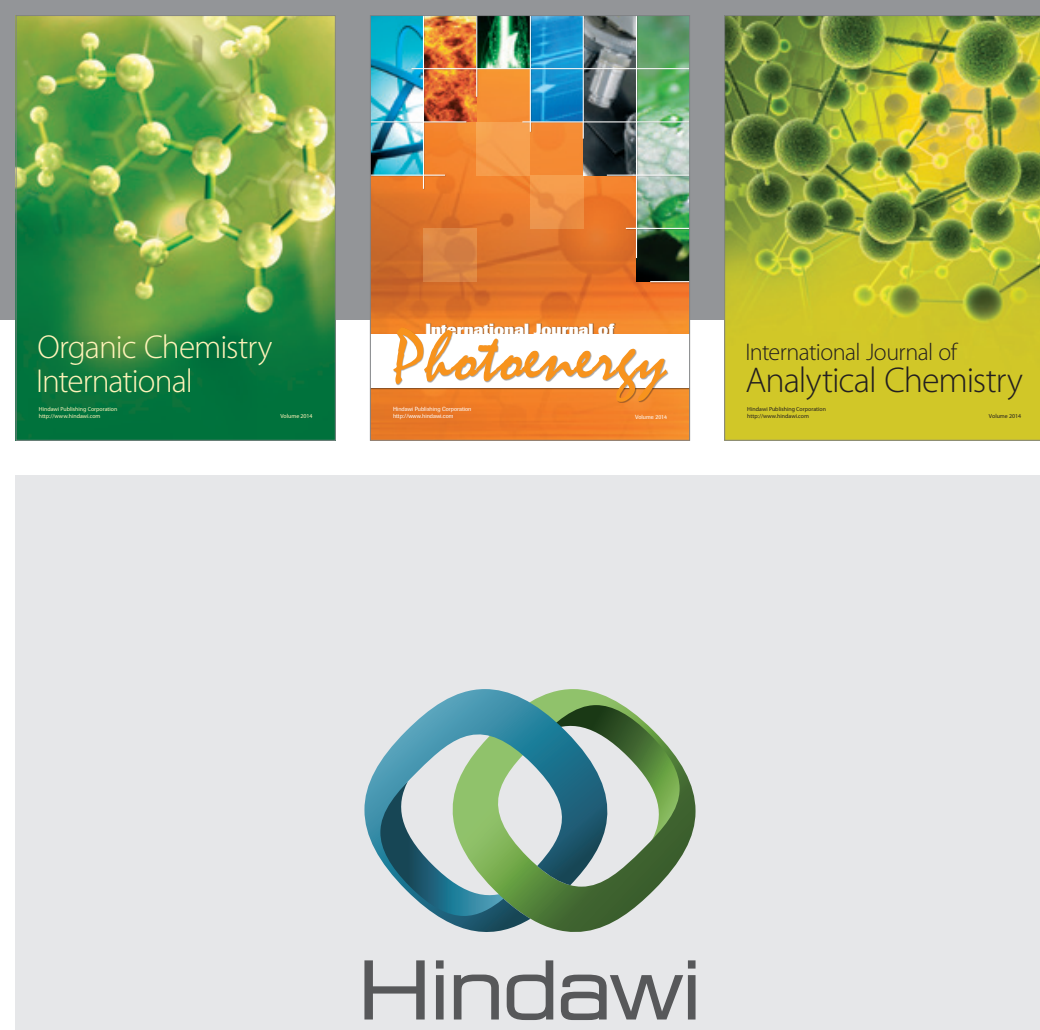

Submit your manuscripts at

http://www.hindawi.com
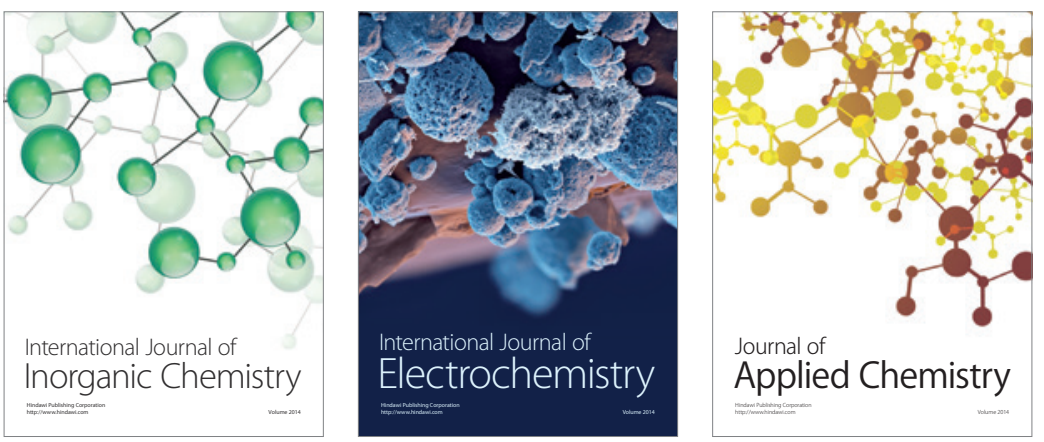

Journal of

Applied Chemistry
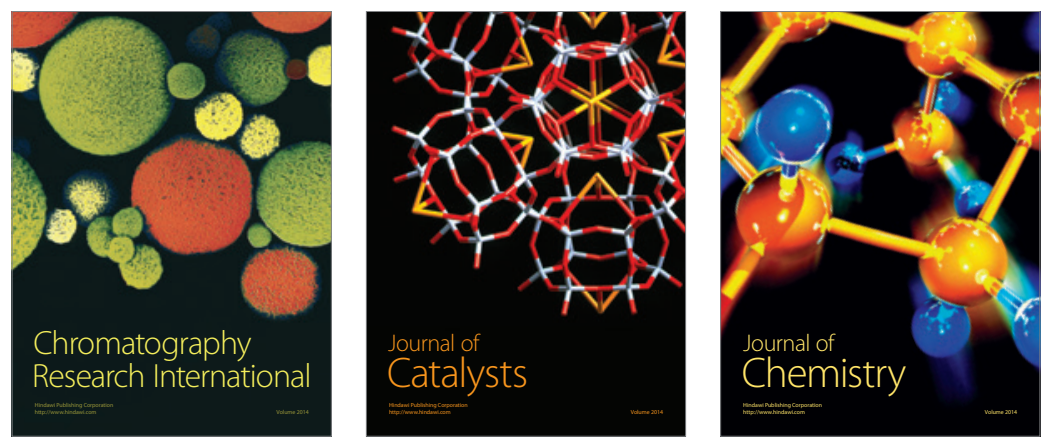
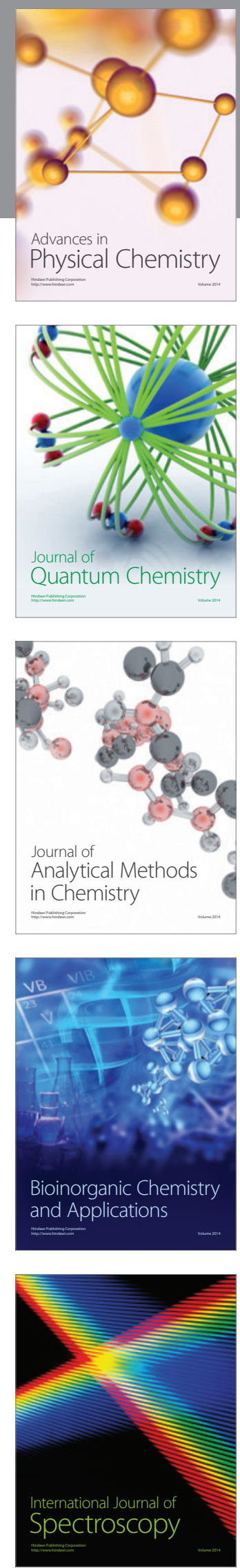